\title{
ORDINE DELLA NATURA E FIGURE DELLA VERITÀ
}

\author{
CARLO SINI (*)
}

SuNTO. - La conoscenza umana e l'ordine della natura: il problema della loro relazione è il centro del contributo. La soluzione chiama in causa la collaborazione tra conoscenza tecnologico-strumentale e lavoro sociale del linguaggio.

ABSTRACT. - The human knowledge and the order of nature: the problem of their relationship is the core of paper. The solution concerns the co-operation between the instrumental and technological knowledge and the sociale work of language.

«La cosa più incomprensibile nell'universo è la conoscenza»: sembra che così si sia espresso una volta Einstein. In realtà, questo è un problema che ha accompagnato la filosofia sin dai suoi esordi: penso per esempio al Teeteto di Platone. Charles Sanders Peirce, filosofo e scienziato, padre del pragmatismo americano, osservò che la questione della conoscenza impone un postulato e cioè che vi sia una connaturalità tra la mente umana e il mondo. La natura, diceva, evidentemente "feconda" la mente umana, facendovi crescere idee che assomigliano alla loro genitrice: la natura.

In particolare per la conoscenza, l'esperienza e l'induzione sono necessarie, ma non sufficienti. Se il sesso del nascituro può dipendere dalla configurazione dei pianeti o da ciò che succede agli antipodi, la ricerca induttiva diventa impossibile. Essa deve preventivamente rivolgersi a una plausibile area di fenomeni e cercare in essa la soluzione. Ma che quell'area, e non un'altra, sia plausibile si può decidere solo con un atto di insight: circolo vizioso inevitabile che nondimeno governa l'abduzione, cioè il verace metodo della scienza secondo Peirce. Esso con-

(*) Già docente di Filosofia teoretica all'Università degli Studi di Milano, Italia. E-mail: sini.carlo99@gmail.com 
siste nel supporre retrospettivamente uno stato di cose la cui premessa e la cui azione giustificherebbero lo stato della situazione presente. Per esempio: tornando a casa scorgo una cartolina nella mia cassetta delle lettere e ne derivo l'ipotesi che sia passato il postino. La nostra indiscutibile capacità di intuizioni fortunate (come dimostra il successo straordinario dell'impresa della scienza moderna) suggerisce che la mente umana sia affetta da un tropismo verso la verità. Detto altrimenti: noi siamo i girasoli della verità. Quindi, la natura dell'essere umano è strutturalmente connessa alle sue capacità conoscitive e, in a long run, alle imprese della ragione scientifica. Piega del mondo, prodotto della evoluzione naturale, l'azione del corpo senziente e della intelligenza degli umani ha ereditato dalla sua nascita comportamenti dotati di successo, per esempio a differenza di altre specie di ominini (come si dice oggi). Se così non fosse, non saremmo qui a pensarlo e a dirlo.

Questa considerazione riguarda in generale tutte le forme viventi sul pianeta, relativamente al loro adattamento ai rispettivi ambienti ecologici, ma quando si parla di conoscenza umana si intende anche altro e in generale molto di più. Ne è prova ed esempio eloquente l'intuizione che ha ispirato la costruzione della tavola periodica degli elementi (una tipica "abduzione") e il suo perdurante successo. Ne deriva, per la mentalità comune (inclusa quella scientifica), che noi non dubitiamo, da un punto di vista chimico, che il mondo sia fatto cosi come la tavola dice e predice. Ma come deve essere fatta la mente umana per arrivare a intuire la verità della natura? Qui cade la tentazione di Galileo: possiamo conoscere la verità perché la nostra mente è, per creazione, simile a quella di Dio, il creatore; naturalmente entro limiti ragguardevoli e in un cammino infinito. Anche Einstein, del resto, sosteneva che Dio non gioca a dadi. Purtroppo queste considerazioni, come ebbe a obiettare proprio Peirce, sono insignificanti. Meritano rispetto, diceva Peirce, perché mostrano di aver compreso la profondità del problema; nel contempo però non servono a niente, perché non spiegano alcunché.

Un tentativo più promettente (che qui mi limito a tratteggiare per cenni molto sommari) suggerisce che il passaggio dalla intelligenza animale a quella umana sia favorito e infine reso possibile dalla natura intermedia dello strumento. Per esempio l'uso di un ramo d'albero come bastone. Esso è un prolungamento esosomatico del braccio, ma non è il braccio; inoltre proviene dal mondo, ma non è più semplicemente mondo, sua parte vivente. Ora, è proprio il medio, il mezzo, ciò che sta in mezzo, a rispecchiare all'agente la natura inconsapevolmente stru- 
mentale del suo stesso corpo in azione, che è contemporaneamente corpo vivente (in tedesco Leib) e corpo-cosa (Körper). Nel medio l'agente impara a leggere il fine stesso dell'azione vivente, analizzandone gli aspetti, vissuti dapprima nella unità dell'azione: che cosa fa la spalla, il gomito, il polso ecc. Rendendo l'azione analiticamente trasparente, il bastone educa il braccio. Nel contempo mostra anche i limiti dell'azione braccio-bastone: il ramo-bastone oppone per suo tramite all'azione l'inerzia originaria del mondo e così mostra che cosa "oggettivamente" si può fare nel mondo con i bastoni e che cosa non si può fare. Quindi suggerisce la necessità di altri strumenti esosomatici ad hoc, inaugurando il cammino infinito della intelligenza tecnica, che fa tutt'uno con l'intelligenza della natura umana, rispetto alla natura animale: l'essere umano è costitutivamente un essere "tecnico" e ciò che chiamiamo "cultura" è letteralmente una macchina, ovvero un automa (qualcosa che già si muove da sé, prima delle intenzioni umane, quelle intenzioni che lo strumento tecnico, appunto, e-duca, analizza e trae fuori allo scoperto).

Manca però un ulteriore, indispensabile tassello, rappresentato da quello strumento e medio essenziale che è, per dirla in modo molto generico, il linguaggio. Anch'esso, si badi, è uno strumento esosomatico, caratterizzato dalla peculiarità della voce, cioè dal suo risuonare "nel mezzo" e tornare indietro autorispecchiandosi nel locutore, che solo ora si sa tale, come appunto solo il gesto della voce sa fare. La voce innesca così la risposta che, disse George Herbert Mead, indica a ogni agente umano che cosa si è pronti a fare in comune e quindi il significato specifico veicolato da ogni gesto vocale. Di qui la nascita in ogni locutore dell'autocoscienza e della nozione di comunità intersoggettiva socialmente operante.

Questa azione intrecciata, variegata e complessa dello strumento esosomatico puro e semplice e di quello strumento che è il linguaggio ricompone ai nostri occhi l'unità profonda della esperienza umana e il senso "vero" del suo conoscere, liberando nel contempo la concezione scientifica della conoscenza e della verità da supposti misteri e soprattutto da un superstizioso riduzionismo "naturalistico". In ogni momento l'oggettività e universalità delle conoscenze è garantita e insieme delimitata dall'uso specificamente strumentale-tecnico della società umana al lavoro, sempre nelle circostanze e relazioni storicamente determinate della sua esistenza. Nel contempo la produzione e l'azione strumentali sono circoscritte e attraversate dalle esigenze, intenzioni, progetti, speranze, credenze di un gruppo umano costituitosi in base alle conse- 
guenze sociali dei suoi discorsi intersoggettivi. Conoscenze e credenze lavorano insieme, secondo figure specifiche e transeunti della verità.

Per esempio noi possiamo trovarci d'accordo nel dire: la storia delle conoscenze umane rivela un costante progresso. Abbiamo molte buone ragioni per pensare così, ma non è una buona ragione dimenticare nel contempo il lavoro che è sotteso alla costruzione di un enunciato siffatto. Non stiamo per niente enunciando una "verità oggettiva in sé" della "natura" e "dell'uomo". Non sono mai esistiti oggetti in sé come la storia delle conoscenze, la conoscenza in generale, il progresso, l'uomo e simili. Questi termini hanno legittimo e condiviso senso solo per noi, abitatori di un certo mondo del sapere e del fare, del conoscere e del dire. Non ha invece senso retrofletterli presso umanità che vissero, o ancora in parte vivono, altrimenti: per tali umanità non è possibile concepire e costruire enunciati come quelli di cui sopra. Non pensava e non sperimentava la vita e il mondo così neppure Francesco Bacone, figuriamoci Lucrezio o Epicuro.

La verità della natura cammina unitamente alla verità esperita e costruita in ogni tempo dal complessivo lavoro umano, strumentale e verbale: noi e le nostre conoscenze e credenze non facciamo eccezione. La verità non è una "cosa", un oggetto o l'astratto contenuto di una proposizione, ma è un destino infinito, iscritto, come diceva appunto Peirce, in a long run. Nel nostro tempo il lavoro della conoscenza è una scommessa abduttiva che organizza il nostro stare e avere il mondo secondo un orizzonte di senso che le sempre nuove e straordinarie conoscenze scientifiche sollecitano alla riflessione, provocando, mettendo in questione, la consistenza delle nostre credenze. In questo senso la conoscenza e l'ordine della natura sono nozioni della reale comunità vivente, cioè della attuale polis e del suo lavoro sociale, liberato da pregiudizi naturalistici e aperto al transito delle figure di verità che la determinatezza della nostra vita collettiva produce e sperimenta.

\section{BIBLIOGRAFIA}

George H. Mead, Mente, Sé e Società, trad. it, Barbera, Firenze 1966.

Charles S. Peirce, Collected Papers, Harvard University Press, Cambridge (Mass.) 1960. Carlo Sini, Inizio, Jaca Book, Milano 2016. 